\title{
Scripta
}

Revista Internacional de Literatura i Cultura Medieval i Moderna

\section{Incerta glòria di Joan Sales tra filologia, storia e traduzione}

Incerta glòria of Joan Sales, between philology, history and translation

PAtrizio Rigobon rigobon@unive.it

Università Ca'Foscari di Venezia

Riassunto: In questo saggio si fa una riflessione sull'opera di Joan Sales Incerta glòria e particolarmente si vede le conessione tra filologia, storia e traduzione.

Parole chiave: Joan Sales, Incerta gloria, traduzione, storia, filologia

Abstract: This article makes a reflection on the work of Joan Sales, Incerta glòria, and it particularly affects the connection between philology, history and translation.

Keywords: Joan Sales, Incerta gloria, philology, history translation 
Joan Sales risulta essere un autore particolarmente idoneo a una riflessione che coinvolga le complessità della storia spagnola del XX secolo, non disgiungendole dalla precarietà della vita letteraria catalana e della sua conoscenza al di fuori dei limiti nazionali (catalani e spagnoli) durante il franchismo. Nel suo romanzo Incerta glòria, la guerra civile, uno degli eventi fondamentali di quel Novecento, Sales entra in tutti gli aspetti, politici, civili, etici ed esistenziali del conflitto, con un punto di vista certamente chiaro, quello dei perdenti, ma senza ideologismi preconcetti. Vi entra la guerra civile, ma anche il regime che ne è seguito; vi entra l'esperienza dell'esilio che l'autore visse in Messico fino al 1947, prima di rientrare in Spagna e diventare, oltre al grande scrittore che abbiamo conosciuto, il principale editore di narrativa catalana, in un momento in cui la vita stessa di quella lingua era stata messa in discussione dal regime franchista. Fu un rientro doloroso, carico di difficoltà e di silenzi a cui lo scrittore fu costretto, dichiarandosi addirittura fortunato perché, come scrisse al suo editore francese (Gallimard) in occasione della traduzione in questa lingua di Incerta glòria: «se devait déjà considérer assez heureux qu'on ne l'eut pas fusillé» (Pla 2002: 542). In queste condizioni non poteva essere un'impresa facile scrivere un romanzo in cui la guerra civile costituisse il motore, lo sfondo, il fine delle azioni dei tre principali protagonisti, Lluis Brocà, Juli Soleràs e Cruells. Infatti la storia testuale è quanto mai accidentata e vedremo di ricostruirla per sommi capi. Un esaustivo lavoro filologico, secondo criteri scientifici stringenti, in questo senso deve ancora essere fatto, malgrado numerosi interventi puntuali, anche di notevolissimo livello, su questo o quell'aspetto (Garcia Raffi 2012). Uno studio filologico sulla materialità del testo, nella cui formazione convergono elementi esterni ed interni, a cui pure le traduzioni, pubblicate o inedite, hanno certamente contribuito, è ancora di là da venire. Sintetizzando, l'azione censoria in primis, ma anche un continuo ripensamento del romanzo, che è andato maturando con il progredire del tempo e il perfezionarsi dell'idea letteraria che lo sorregge.

Paola Italia nel suo bel volume Editing Novecento sostiene che «se l'autore di un'opera è un soggetto unico, l'autore della sua realizzazione editoriale è un soggetto multiplo, che comprende almeno tre figure: 1) l'autore vero e proprio, 2) il curatore della sua opera, 3) il redattore che s'incarica di seguirne tutti i passaggi redazionali» (Italia 2013: 13-14). Aggiungerei che, nel caso di molti autori, della seconda metà del Novecento in particolare, un peso notevole hanno le mogli o vedove che configurano un soggetto che assume su di sé assai spesso i ruoli dell'autore (se defunto), del curatore certamente e, spesso, anche del redattore editoriale. Nel caso che qui c'interessa Núria Folch Pi, la vedova dell'autore morta nel 2010 a 94 anni, ha rivestito certamente tutti e tre questi ruoli, anche per una notevolissima esperienza professionale nell'ambito dell'editoria e una comunione e contiguità esemplari con l'autore. Sgombero il campo dagli equivoci: non voglio certo sminuire un ruolo che è fondamentale nel mantenimento della memoria, nella custodia dei materiali critici e nella loro utilizzazione e nella definizione di una verità storica, tanto più che la persona in questione ha sempre collaborato con tutti gli studiosi dell'opera del marito, appare tuttavia evidente che la prossimità familiare può determinare qualche, chiamiamola così, «aporia filologica». C'è poi l'editore Sales, un imprenditore, che certamente ha un peso sull'autore Sales, che tende a moltipilcare le «edizioni» per enfatizzare il successo di un'opera, anche se spesso si tratta di ristampe. Così scrive 
a Mercè Rodoreda a proposito del El carrer de les Camèlies: «Sobre l'edició en offset del Carrer ja us deia a la meva anterior la solució finalment adoptada [...]: en fem 4000, però d'ells, 2000 sense les primeres i últimes pàgines (aquelles on figuren el número d'ordre i la data de l'edició), de manera que la $3^{\text {a }}$ edició constarà de $2000 \mathrm{i}$, quan els haurem despatxats, els altres 2000, completats amb aquelles pàgines, que tirarem aleshores, constituiran la $4^{a}$ edició» (Rodoreda \& Sales 2008: 338). ${ }^{1}$

Prima dientrare nel merito della storia testuale, crediamo opportuno ricordare alcuni dati. La redazione del romanzo comincia nel 1948, l'anno successivo il rientro a Barcellona dall'esilio messicano: ne vediamo la prima edizione a stampa nel dicembre del 1956, conseguenza della vittoria in un prestigioso premio letterario, il Joanot Martorell nel 1955. Questa edizione ha 335 pagine. Su questa si è in particolare esercitata la censura. Il rapporto sul romanzo stilato dal «lector núm. 32» recita: «Novela escrita bajo la forma de diario de un oficial del Ejército rojo durante la guerra de España. Expresa ideas heréticas -las propias de semejante individuo- y a menudo en un lenguaje grosero y asqueroso. Se desprende una filosofía existencialista, condenable por la forma y el fondo. Las ideas condenables constituyen el verdadero fondo de la novela; de manera que, aunque se suprimieran pasajes enteros, la obra seguiría siendo absolutamente impublicable. Es preciso prohibirla en absoluto». ${ }^{2}$ Non molto distanti le altre schede di lettura per cui le forbici hanno presumibilmente «sfoltito» parecchio il testo, fino a renderlo pubblicabile. Questo, in realtà, non coincide con quanto afferma Núria Folch la quale afferma: «Si bé és vera veritat que és reduïda a causa del'existència de la censura i de la repressió, no ho és que hagué estat 'mutilada per la censura', com deixà creure i abonà que es cregués Joan Sales. [Ell] aniria progressant en l'art d'esquivar-la» (Sales 2010: 12). ${ }^{3}$ Più che affrontarla, a Sales sembrava pragmaticamente più produttivo eluderla. Ma a quale prezzo e con quali riflessi sul contenuto delle opere? Certo esercitandosi, probabilmente come molti altri in quel periodo, nell'arte dell'«autocensura», come conferma Carlos Pujol, traduttore spagnolo di Incerta glòria: «però hi havia la qüestió espinosa que no era fàcil que aprovessin i publiquessin la novel la. En vaig parlar amb l'editor, i ell em va dir que em feia responsable del que podia passar. Quan li vaig plantejar la qüestió a Sales, vam concordar que treballariem junts per suavitzar-ne algunes escenes o expressions que poguessin cridar l'atenció de la censura» (Pujol 2011: 16). A dire il vero Núria Folch $(I G, 2010,11)$ parla anche di «tirania suavitzada per la corrupció» che rendeva

1 Per essere ancora più chiari, Sales si riferisce anche alla moglie in merito al vantaggio delle «edicions curtes»: «[...] la meva dona [...] és com si diguéssim més papista que el papa o sigui més partidària que jo mateix del meu mètode de les edicions curtes, que si bé no resulten gaire econòmicament en canvi tenen l'avantatge de la ressonància psicològica d'allò de: $2^{\mathrm{a}}$ edició, $3^{\mathrm{a}}$ edició, $4^{\mathrm{a}}$ edició, que hem anat veient que era la propaganda eficaç, l'única en què el públic creu» (Rodoreda \& Sales 2008: 338).

2 Cit. nel blog di Club editor senza riferimento a una fonte precisa pubblicata o inedita <http://editor1984. blogspot.it/2010/07/impublicable_09.html>, consultato il 23 gennaio 2015. Cit. anche Francesc-Marc Alvaro, «A Frankfurt con Joan Sales» in La Vanguardia (Barcelona), 17 de septiembre de 2007, p. 20.

3 Citeremo nel testo quest'edizione con la sigla «IG, 2010», seguita dall’indicazione della pagina. Stando al verso del frontespizio di questa edizione, si tratta della «Tercera reimpressió: juny del 2010» (verosimilmente) della «segona edició definitiva en aquesta col lecció: 1999».

SCRIPTA, Revista internacional de literatura i cultura medieval i moderna, núm. 5 / juny 2015 / pp. 281 - 291 ISSN: 2340-4841 · doi:10.7203/SCRIPTA.5.6395 
l'ostacolo censorio in qualche modo aggirabile, non entrando tuttavia in ulteriori dettagli. Da osservare che, in realtà, la radice cattolica dell'autore, potenzialmente meritevole della benevolenza del nazionalcattolicesimo franchista (peraltro da lui detestato), è chiaramente percepibile in questo testo, dove appare anche la dimensione esistenziale in un contesto filosofico e morale debitore di tanta letteratura europea, in particolare di quegli scrittori cattolici più versati all'eterodossia; dato che, evidentemente, non ha mitigato il giudizio del censore citato. Si tratta di un'intertestualità certa, perché alcuni di questi scrittori Sales li aveva o li avrebbe tradotti in catalano e pubblicato con la sua casa editrice. Inoltre, nella rivista che a Città del Messico aveva fondato e diretto dal 1943 al 1947, Quaderns de l'exili, l'elaborazione di un catalanismo problematico di matrice cattolica è molto evidente e sancisce l'approdo di un sofferto passaggio morale e ideologico (l'autore negli anni Trenta aveva militato, sia pure per poco, nel Partit Socialista Unificat de Catalunya d'ispirazione comunista) che la guerra civile aveva già contribuito ad orientare, come chiaramente si evince anche dal romanzo. La complessità della questione potrebbe essere rappresentata dal personaggio di Juli Soleràs, molto vicino al sentimento dell'autore non soltanto per la prossimità fonetica dei due nomi, che presenta tratti di scetticismo che rasentano il cinismo. Ma le articolazioni di un cattolicesimo complesso sono evidenziate anche dall'ex seminarista Cruells e dal protagonista della prima parte, Lluís Brocà. Torniamo però alla storia esterna del romanzo che inizia dunque nel 1956, momento in cui una visione della guerra civile da un angolatura diversa da quella dei vincitori del conflitto, rappresentava di per sé uno scoglio rilevante. Ricordiamo che uno dei testi ufficiali, per così dire, del canone storiografico franchista sull'argomento, i volumi della Historia de la Cruzada española di Joaquín Arrarás, preferiscono appunto evidenziare l'aspetto della lotta contro l'infedele che la parola «Crociata» sottende, sinonimo quasi di «Reconquista», che sublima l'aspetto del conflitto ideologico, così come l'abbiamo conosciuto nel Novecento, in un evento di lunga durata. La storia del testo comincia dunque sotto il pesante giogo della censura. Un'ulteriore edizione, non già una semplice traduzione, sarà quella pubblicata a Parigi da Gallimard sulla base di un rapporto di lettura di Juan Goytisolo che viveva allora Parigi. Xavier Pla ha ricostruito dettagliatamente la storia di questa traduzione che è in realtà una nuova edizione. Molto più estesa dell' edizione del 1956 con un processo redazionale articolato perché il traduttore, l'occitanista Bernard Lesfargues, riceveva il materiale in dattiloscritto man mano che veniva prodotto. Ciò evidentemente significava che non esisteva una versione del 1956 definitiva, quella presumibilmente in possesso dell'autore e pubblicata solo in parte in Catalogna, ma una redazione ancora in atto con cambiamenti, rimaneggiamenti e aggiunte. Lo conferma Núria Folch: «Bernard Lesfargues aniria traduint a mesura che Joan Sales l'anava, en part, reescrivint, afegint-hi coses encara impublicables a Espanya» $(I G, 2010,13)$. Cioè - deduciamo - ben più delle parti omesse, volontariamente o involontariamente, nel 1956. L'edizione francese fu un successo di critica e di pubblico che mise seriamente in imbarazzo il regime tanto che all'autore venne immediatamente ritirato il passaporto. Interessante la valutazione di un recensore, Jacques de Ricaumont, che sottolinea come l'autore cercasse la verità «lungi dalla menzogna nera e da quella rossa» (cit. in $I G, 2010,13$ ). Porre la questione del giudizio storico di una «terza via» per un autore che era stato, e continuava ad essere, decisamente repubblicano, costituiva 
una relativa novità. Non ho detto «terza Spagna» perché in realtà Joan Sales si è sempre considerato combattente della Catalogna, e più specificamente dell'esercito catalano repubblicano.

Quattro anni dopo l'edizione francese, Rizzoli, sull'onda del successo avuto in Francia, acquisisce i diritti di traduzione in italiano e affida il lavoro a una giovane ispanista, Mariateresa Cattaneo. Su questo abbiamo una sua recente testimonianza, fondamentale per ricostruire l'episodio, visto che la traduzione italiana non vide mai la luce: anche in questo caso la fonte delle parti tradotte (la prima e quasi tutta la seconda) era costituita da dei fogli dattiloscritti che venivano inviati man mano perché l'autore «treballava constantment sobre el text, la qual cosa evidenciava la seva recerca obstinada d'una prosa catalana fluïda i viva, col loquial i elegant al mateix temps, amb les seves inflexions populars, la riquesa de la tradició i el seu caràcter peculiar.» (Cattaneo 2011: 22).Va detto che la traduzione partiva dalla versione francese, anche se era stato inviato all'autrice l'edizione catalana (del 1956) per avere contezza dello stile e della prosa originali. Svolta buona parte del lavoro, l'autore chiese all'editore e alla traduttrice di fermarsi in attesa della versione integrale a stampa. Quando questa usci, nel 1969, spaventò per lo spessore la Rizzoli che, avendo cambiato nel frattempo il direttore editoriale, decise di rinunciare all'impresa. La questione della versione non completata e, comunque, non pubblicata, riveste un duplice interesse, perché, da un lato, conferma la modalità di lavoro sul testo da parte dell'autore, configurandolo quasi come un «work in progress», dall'altro le difficoltà a pubblicare letteratura catalana in Italia (ma non solo) in un periodo in cui la Spagna, a causa principalmente del regime franchista, si trovava in una emarginata periferia ideologica a cui solo la guerra fredda poteva attribuire una qualche «utile» funzione. Di questa parziale traduzione, consegnata dalla traduttrice all'editore, non esiste traccia nell'archivio storico Rizzoli, secondo quanto ci ha confermato la responsabile del medesimo, Francesca Tramma. In attesa di ulteriori accertamenti archivistici, della consuetudine che si era stabilita, a metà degli anni Sessanta, tra Mariateresa Cattaneo e Joan Sales, rimane solida traccia anche nel suo epistolario con Mercè Rodoreda. Addirittura qualche stralcio del contratto sparito (o non presente) negli archivi dell'editore milanese. In una lettera alla Rodoreda, Sales riporta di quel contratto per la traduzione del suo Incerta Glòria con Rizzoli le condizioni economiche legate al numero di copie vendute. Citiamo così com'è riportato nell'epistolario:«un droit de $8 \%$ sur le prix fort pour les premiers 5000 (CINQ MILLE) exemplaires, et 10\% au-delà de 5000, de l'édition brochée» ${ }^{4}$. Per quanto riguarda la lingua da cui traduceva, Mariateresa Cattaneo dichiara che «es tractava bàsicament de traduir del francès: la voluminosa edició de Gallimard de Gloire incertaine (traducció de Bernard Lesfargues) constituïa el text, al qual s'havia d'afegir un petit llibre en català, que era tot el que la censura franquista havia permès que es publiqués, i que em podia servir com a ajuda» (Cattaneo 2011: 21). Interessante la riflessione di Sales sulla lingua da cui tradurre il suo romanzo (e quello della Rodoreda) che prescinde da criteri filologici sulla lingua fonte ed enfatizza l'importanza della lingua d'arrivo. Scrive infatti Sales all'autrice de La Plaça del Diamant: «Respecte a la consulta que fan, de si han de traduir del català o del castellà, jo us aconsellaria de respondre: que ho facin traduir

4 Lettera 150, del 3 febbraio 1966 (Rodoreda \& Sales 2008: 269).

SCRIPTA, Revista internacional de literatura i cultura medieval i moderna, núm. 5 / juny 2015 / pp. 281 - 291 ISSN: 2340-4841 · doi:10.7203/SCRIPTA.5.6395 
del castellà però facin veure que ho han fet del català (és així, més ben dit del francès, que ho fan amb Incerta glòria a can Rizzoli) perquè si us poseu farruca que cal traduir directament del català, ho daran a traduir a alguna rata de biblioteca, filòleg i pedant, que tindrà quatre nocions de català arcaic, i farà una traducció italiana encarcarada i probablement absurda. Mentre si ho poden traduir del castellà, ho daran a algun traductor de qui ja sàpiguen que té brio i salero a escriure en italià -que és el principal- encara que no hagi fet mai fitxes sobre la freqüència del 'llur' i del 'quelcom' en les obres juvenils d'Eiximenis comparades amb les de Bernat Metge, ni hagi mai sentit parlar de Ramon Llull». ${ }^{5}$ In una lettera del 3 marzo 1966 Joan Sales annuncia alla Rodoreda che «a darreries d'aquest mes ve a passar un parell de setmanes a Barcelona la meva traductora italiana, i també ho aprofitaré per parlar-li de la Plaça i obsequiar-li un exemplar català i un altre castellà, perquè en recomani la publicació a Rizzoli». ${ }^{6}$ In una successiva lettera, insieme al capolavoro della Rodoreda, riesce a dare anche un giudizio deciso sulla qualità letteraria di una notissima autrice barcellonese di lingua spagnola: «La Mariateresa Cattaneo (la meva traductora italiana) ja se n'ha anat enduentse'n La Plaza del Diamante. [...] [L’] únic que em preocupava és assegurar-me que la llegiria, perquè estic cert que si la llegeix, la proposarà a Rizzoli. Ella vinga a parlar-me de la Matute, i jo vinga a fer equilibris per fer-li entendre que la Matute no és res - en un cert moment vaig arribar a dir-li que era 'coca-cola' - al costat de vós». ${ }^{7}$ Tuttavia è in una successiva missiva del 17 settembre 1966 che l'editore, escogitando un disegno «machiavellico» per tenere aperte le possibilità sia con Mondadori che con Rizzoli di pubblicare l'opera maggiore della «sua» Rodoreda, esprime una preferenza per la seconda casa editrice perché «hi ha la Mariateresa que sap el català (és professora de llengua i literatura castellana i catalana a la universitat de Milà) i que està traduint Incerta glòria molt bé (me’n va ensenyar uns llargs fragments, de mostra, divinament traduits). I és allò de: més val boig conegut que savi per conèixer». ${ }^{8}$ Per la traduzione italiana d'Incerta glòria, la metodologia di lavoro fu dunque simile, per molti aspetti, a quella collaudata con Bernard Lesfargues: non si partiva da un testo già pubblicato (in questo caso nemmeno dalla versione francese che poteva essere la più completa), ma da nuove cartelle dattiloscritte: «Així vam començar a fer la traducció: Sales em portava cada setmana un feix de papers a màquina, i de fet non vaig veure mai cap pàgina impresa, ni de l'edició francesa ni de l'anterior catalana. I la setmana següent jo li lliurava la traducció i ell em portava l'altra part de la novel la. D'aquesta manera, el llibre s'anava traduint a mesura que ell l'escrivia» (Cattaneo 2011: 26). Per quanto riguarda la versione originale catalana, a cui dunque l'autore aveva ripetutamente messo mano rispetto al testo del 1956, Sales annota significativamente, prendendo spunto dall'autorizzazione della censura alla pubblicazione della traduzione spagnola di Carlos Pujol, «Incerta glòria ha arribat totalment aprovat de censura en castellà, cosa que cal atribuir a la

5 Lettera 173 del 19 febbraio 1967 (Rodoreda \& Sales 2008: 330).

6 Lettera 156 del 3 marzo 1966 (Rodoreda \& Sales 2008: 287).

7 Lettera 157 del 13 aprile 1966 (Rodoreda \& Sales 2008: 290).

8 Lettera 158 del 17 settembre 1966 (Rodoreda \& Sales 2008: 292).

SCRIPTA, Revista internacional de literatura i cultura medieval i moderna, núm. 5 / juny 2015 / pp. 281 - 291 ISSN: 2340-4841 · doi:10.7203/SCRIPTA.5.6395 
influència de Lara [...] [i] que hagin aprovat íntegrament l'edició castellana d'en Lara ens permet a nosaltres llançar també íntegrament la catalana».

Nel 1969 uscì dunque quest'edizione catalana, quasi in contemporanea con la traduzione castigliana di Carlos Pujol, ma è (sono) veramente integrale ovvero «definitiva»? Certamente no, secondo Núria Folch: «definitius es declareren doncs encara que no fos cert» $(I G, 2010,15)$. Ma cos'era accaduto? La censura s'era improvvisamente rilassata? Evidentemente no, tuttavia qualcosa era cambiato. Il 19 marzo del 1966 era stata pubblicata, per iniziativa dell'allora ministro Manuel Fraga Iribarne, la «Ley de Prensa e Imprenta» che, agli articoli 3 e 4 eliminava la censura previa e la «consulta obligatoria», introducendo un nuovo istituto la «Consulta voluntaria»: «La Admnistración no podrá aplicar la censura previa ni exigir la consulta obligatoria, salvo en los estados de excepción y de guerra expresamente previstos en las leyes.

Artículo cuarto. [...]. La Administración podrá ser consultada sobre el contenido de toda clase de impresos por cualquier persona que pudiera resultar responsable de su difusión. La respuesta aprobatoria o el silencio de la Administración eximirán de responsabilidad ante la misma por la difusión de impreso sometido a consulta». ${ }^{10}$

La censura rimane, ma viene, per così dire, spostata a valle. Qui le ricostruzioni di Núria Folch e Carlos Pujol divergono leggermente. Per la prima, «més valia doncs als 'sospitosos' editors d'obres catalanes, presentar-les a la dita Consulta Voluntaria» (IG, 2010, 14), mentre Pujol dice che: «Mentrestant ell [Sales] tenia preparada l'edició catalana atès que la volia treure pocs dies després de l'edició castellana, sense demanar cap permís, al ludint que ja existia un permís per la traducció». A questo si aggiunge il «modus operandi» nella redazione della traduzione, analogo a quello descritto dalla traduttrice italiana ( Sales em portava cada setmana un feix de papers a màquina, i de fet no vaig veure mai cap pàgina impresa») (Pujol 2011: 26). In ogni caso, come ricorda Pujol, nel 1970 venne dichiarato un «estado de excepción»che ripristinò la censura previa e quindi il libro venne inviato alla censura che, sorprendentemente, non toccò una virgola. Perché era successo questo? Rispondere alla domanda significa anche entrare nel merito del testo, proprio in vista del suo esame, da parte della censura: non sempre è facile distinguere ciò che è stato cambiato proprio per superare quest'esame oppure per una consapevole, ma libera, scelta dello scrittore. Proprio il rapporto traduttore-autore nella versione castigliana ce lo fa chiaramente capire. Scrive Pujol: «quan li feia observacions de cara a la censura [Sales] les acceptava sense dir-me absolutament res. Cal dir que una vegada publicada l'edició castellana, en edicions successives ell va anar afegint i restablint a la catalana tot el que haviem suavitzat o eliminat per la censura» (Pujol 2011: 26)

9 Lettera 195 del 21 marzo 1969 (Rodoreda \& Sales 2008: 383). Da ricordare che, esattamente un mese prima, Sales scriveva alla Rodoreda: «El pitjor de tot és que em diu el cor que aquesta feinada de boig no haurà servit per res i que Incerta glòria haurà de seguir tan inèdita, en el seu text íntegre, com fins ara.» (Lettera 194 del 21 febbraio 1969; Rodoreda \& Sales 2008:379).

10 BOE, n. 67, 19 de marzo de 1966. 
Dunque qual è la versione definitiva? Stando a Núria Folch è la quarta edizione, pubblicata nel 1971 nella collana del «Club dels Novel listes» a rappresentare veramente il «text definitiu». Ma è proprio così? Non sembrerebbe, dieci anni più tardi infatti, nel 1981, pubblicata da un circuito editoriale che si chiamava Grup del Llibre riservato ai sottoscrittori, compare la quinta edizione, l'ultima curata dallo stesso autore e quindi, a rigore, «definitiva». Con quale cambiamento? Una piccola, ma poi non tanto, novità. La quarta e ultima parte dell'opera, intitolata nelle prime edizioni, là dove presente, Últimes noticies, quella cresciuta da un'edizione all'altra in modo significativo, viene ora trattato come romanzo «a sé stante», con titolo diverso. Nel frontespizio infatti si legge Incerta glòria seguit d'El vent de la nit. Così giustificato da Sales: «...simplement he decidit seguir el consell insistent de Carles Pujol, traductor insuperable i crític penetrant d'aquesta novel la, fent allò que sota el títol d'Últimes notícies hi apareixia com a darrera part hi aparegui ara, sota el títol d' El vent de la nit, com el que és en realitat una altra novel la, la novel la d'un sacerdot català avançant a palpes a través de la llarguíssima nit» (IG, 2010, 16). Si trova tuttavia nello stesso volume. Cosa però che non accade nello stesso anno in una collana di grandissima diffusione, la MOLC curata dal recentemente scomparso Joaquim Molas che pubblicò il romanzo in due volumi (Incerta glòria I e II), non menzionando però in copertina il titolo El vent de la nit e sopprimendo un' importante pagina iniziale dal titolo «Confessió de l'autor». In ogni caso s'introducono numerosi cambiamenti rispetto all'edizione presumibilmente definitiva del 1971.

La settima edizione (1983) d'Incerta glòria e terza d' El vent de la nit è quella curata dalla vedova. L'opera sta in un solo volume con una distinzione netta tra i due romanzi.

L'ottava e nona edizione (1988 e 1991) sopprimono nuovamente la paginetta «Confessió de l'autor», datata «Barcelona, desembre de 1956, assente fin dalla prima edizione di quell'anno, pubblicata da Aymà. ${ }^{11}$ In questa «confessione» l'autore spiega la scelta del frammento del verso shakespeariano del titolo, ricollegandolo al baudelairiano «ma jeunesse ne fut qu'un ténébreux orage» che rimanda alla ormai lontana gioventù di guerra e d'amore, alla consapevolezza dì essere peccatori assetati di gloria. Una breve pagina, molto densa, che offre certo una fondamentale chiave di lettura del romanzo.

Arriviamo dunque della decima edizione (che ho utilizzato per questo scritto): si tratta di una ristampa del 2010 dell'edizione del 1999: «com ja he explicat, reprodueix el text, els títols i la distribució de l'última edició feta per Joan Sales mateix, la desaparcebuda i ara exhauridíssima cinquena edició» $(I G, 2010,19)$. Ma attenzione, se leggiamo nella pagina dei copyright, citata testualmente nella nostra nota 6, questa versione viene definita un po' diversamente: «Seconda edizione definitiva in questa collana: 1999. Terza ristampa: giugno 2010». Quante edizioni «definitive» ci sono? Qual è

11 Joan Sales, Incerta glòria, Barcelona, Aymà, 1956, p. 335. Questa edizione è certamente interessante, oltre per quello che non ha rispetto alla successive, anche per quello che ha (e manca invece ad altre), come per esempio la dedica della p. 9 (non numerata) «a Xavier Benguerel, Domènec Casanovas, Juan Goytisolo, Jaume Gras, Joan Oliver, Joan Roig Gironella, S.I amics, lectors i consellers».

SCRIPTA, Revista internacional de literatura i cultura medieval i moderna, núm. 5 / juny 2015 / pp. 281 - 291 ISSN: 2340-4841 · doi:10.7203/SCRIPTA.5.6395 
la prima di cui quella del 1999 sarebbe la seconda? Quella del 1981 non era pubblicata nella stessa collana e nemmeno dallo stesso editore, ammesso che quella sia la prima. Poi c'è l'altra questione: Joan Sales aveva dichiarato definitivo il testo della seconda edizione (1969) senza che realmente lo fosse, verosimilmente per «capejar la Consulta Voluntària», cioè per aggirare la legge del 1966. Ma quel che è più stimolante, e anche curioso, è che Núria Folch conclude così il lungo preambolo testuale all'edizione «definitiva» da noi utilizzata: «És, en aquest moment, doncs, aquesta desena edició l'única íntegra i realment definitiva, sense cap mutilació» (IG, 2010, 19). La limitazione temporale non sembra escludere quindi che ci saranno nuove edizioni più o meno definitive in futuro. In effetti nel 2007 compare una undicesima edizione di 764 pagine, nel 2012 una dodicesima edizione, che viene ristampata nel 2014 di 541 pagine. Non ho ancora visto direttamente queste edizioni, quindi non posso esprimere alcun giudizio. Osservo soltanto che il concetto di «definitivo» è quanto mai aleatorio e pone interessanti quanto rilevanti problemi ecdotici. Un caso da sottoporre allo stringente vaglio della filologia d'autore che, acquisendo tutti i materiali (impresa non semplice perché molti dei dattiloscritti di Sales sono andati perduti) possa ricostruire geneticamente le varie fasi. Non so in realtà quanta importanza abbia fissare un testo critico «definitivo», che rifletta la canonica ultima volontà dell'autore, peraltro non sempre individuabile con chiarezza. Vorrei in conclusione introdurre un'eresia filologica alludendo al concetto di testo «flessibile», quell'opera, immortalata nelle sue diverse fasi redazionali, che di volta in volta risponde ad esigenze interne ed esterne diverse. Quel testo che comunque è stato licenziato dall'autore il quale ha risposto in modo per l'appunto flessibile alle circostanze oggettive e soggettive, certo lamentando i forti condizionamenti censori del regime (com'è naturale che sia), ma non rinunciando alla pubblicazione. A questo proposito potremmo anche ricordare il fatto, ben studiato dal citato Xavier Pla, che per l'edizione francese, più ampia di quella catalana del 1956, Sales voleva l'imprimatur delle autorità ecclesiastiche francesi, insistendo con Gallimard. Nihil obstat che, paradossalmente, non giunse. Il romanzo di Sales sembra in un continuo stato negoziale, non solo per l'avversa congiuntura storica, ma anche per un' intima esigenza testimoniale e forse anche morale, nonché ovviamente estetica. Il decadimento dell'uso letterario della lingua catalana in epoca franchista corrisponde a una forte «castiglianizzazione» lessicale e sintattica. L'intenso lavoro di «normalizzazione» dell'uso letterario del catalano, era una necessità di cui Sales era ben consapevole e sulla quale lavorò moltissimo con un preciso punto di vista che non lo collocava certo tra $\mathrm{i}$ «puristi». ${ }^{12}$ Ovviamente uno studio genetico sulle varie fasi redazionali del romanzo potrebbe chiarire in modo tangibile le predilezioni linguistiche di Sales e la sua idea di lingua. Bisognerebbe ancora considerare l'epistolario con Màrius Torres che illustra in modo analitico anche alcuni di questi problemi, nonché le lettere scambiate

12 Si veda, a tal proposito, l'articolo di Jordi Cornellà-Detrell (2007: 118): «Sales creu en la genuïnitat del català parlat, i això l'enfronta a una tradició gramatical que per raons històriques observa la llengua parlada amb desconfiança, sempre suspecta de castellanització. Sales, en contra de l'opinió de la majoria de gramàtics i correctors, creu que el parlant no s'ha d'adaptar a la gramàtica, sinó que la gramàtica s'ha d'adaptar al parlant, i per això no fonamenta la naturalitat de l'expressió en l'ús de la normativa vigent, sinó en l'acostament a la llengua parlada». 
con i traduttori (quelle con Lesfargues sono in buona parte disponibili). Vagliare attentamente tutta la produzione precedente, in particolare gli scritti apparsi in Messico durante l'esilio, possono far comprendere le ragioni dell'instabilità testuale come criterio ineludibile. In tal modo potremmo riuscire a chiarire, se non a definire, il rapporto dell'autore col proprio testo. Un rapporto tutt'altro che cristallizzato: in questo senso l'acquisizione e la ricostruzione della documentazione relativa ai diversi stadi redazionali ci potrà dire parecchio, aggiungendo un importante tassello sulla storia della censura nella Spagna franchista. Ma anche sull'alterazione della memoria, letteraria e non, sull'incertezza e la paura che molti scrittori, rientrati dall'esilio, hanno conosciuto in una patria che non riconoscevano più e dalla quale si sentivano ovviamente ancor più esiliati. Paure concrete che, trasferite in un testo, sono diventate esitazioni, reticenze, accomodamenti. Lo scrittore catalano deve inoltre fare i conti con un aggravio ulteriore: le interdizioni della propria lingua e le difficoltà a mantenerne lo standard letterario.

Vorrei concludere con una riflessione su un aspetto dell'opera di Sales che viene per lo più sottaciuto. Non lo credo secondario perché si tratta di una questione che gli stava molto a cuore: il rapporto tra intellettuale e politica, tra letteratura e storia, che a più riprese aveva trattato anche in Quaderns de l'exili. Nel numero 15 del 1945, a proposito dell'indisciplinatezza e della sostanziale anarchia delle milizie catalane (una delle cause principali della sconfitta e del «desastre nacional») aveva scritto: «L'Exèrcit de Catalunya es pot dir que no va existir més que en els documents oficials, ja que en realitat va continuar la indisciplina, com a fruit natural de tantes anyades de prèdiques demagògiques contra la idea del deure i de l'honor militars. Es just assenyalar que en aquesta tasca nefasta, al costat dels demagogs politics, havien brillat els demagogs literaris de tota mena. Una ullada a la trista literatura catalana dels anys anteriors a la guerra (continuada ara a l'exili per l'escola neofloral-regionalista) ens il lustraria molt sobre les causes que van determinar el Desastre Nacional del 1939»r. ${ }^{13}$ Una rivendicazione della letteratura catalana d'impegno che, se da un lato poteva essere stata «disfattista» quando avrebbe dovuto essere costruttiva, avrà in futuro un ruolo notevole nella ricostruzione. Ottica nella quale forse si può leggere anche Incerta glòria: una rivendicazione della letteratura come riflessione ricostruttiva di un'identità nazionale devastata (Sales pensa ovviamente alla Catalogna), una metafora della gioventù destinata, analogamente ai giorni d'aprile shakespeariani, cui rimanda il titolo scelto dall'autore, ad alludere al destino delle persone e della nazione: «The uncertain glory of an April day / Which now shows all the beauty of the sun/ And by and by a cloud takes all away». Una nuvola durata quarant'anni che oscurò non solo la Catalogna, ma l'intera Spagna, che non si limitò a congelare quella gioventù intellettuale, differendola innaturalmente alla fine dei quarant'anni d'esilio interno od esterno, ma cancellò un'intera età dello spirito e della volontà. A quella difficile rifondazione può ancora ambire la letteratura.

13 Quaderns de l'exili, anno III, n. 15, 1945, p. XIII. Raggiungibile all'indirizzo < http:/ / www.cervantesvirtual. com/obra-visor/quaderns-de-lexili--10/html/025b3048-82b2-11df-acc7-002185ce6064_13.htm>, consultato il 24 gennaio 2015.

SCRIPTA, Revista internacional de literatura i cultura medieval i moderna, núm. 5 / juny 2015 / pp. 281 - 291 ISSN: 2340-4841 · doi:10.7203/SCRIPTA.5.6395 
Patrizio Rigobon. Incerta glòria di Joan Sales tra filologia, storia e traduzione

\section{Bibliografia}

Cattaneo, M. (2011), «Vicissituds de la traducció italiana d'Incerta glòria», Quaderns. Revista de traducció, n. 18, pp. 21-23.

Cornellà-Detrell, J. (2007) «Oralitat i escriptura a la literatura catalana de postguerra», Actes del Tretzé col loqui internacional de llengua i literatura catalanes, vol. II, Barcelona,Publicacions de l'Abadia de Montserrat, pp. 117-129.

Garcia i Raffi, J. V. (2012) «Bibliografia de Joan Sales: obra i recepció crítica», Estudis romànics, n. 34, pp. $450-454$.

Italia, P. (2013) Editing Novecento, Roma, Salerno editrice.

$\mathrm{Pla}$, X (2002) «Incerta glòria de Joan Sales o una poètica de l'excés», Estudi general. Revista de la Facultat de Lletres de la Universitat de Girona. Miscel lània d'homenatge a Modest Prats, II, n. 22, pp. 529-553.

Pujol, C. (2011) «La traducció al castellà d'Incerta glòria», Quaderns. Revista de traducció, n. 18, pp. 25-28.

Rodoreda, M. \& Sales, J. (2008) Cartes completes (1960-1983), a cura de Montserrat Casals, Barcelona, Club editor.

Sales, J. (2010) Incerta glòria seguida d'El vent de la nit, Barcelona, Club editor. 